\title{
The Surface Brightness Fluctuations and Globular Cluster Populations of M87 and its Companions
}

\author{
Eric H. Neilsen Jr., Zlatan I. Tsvetanov, Holland C. Ford \\ Johns Hopkins University, Baltimore, MD, USA
}

\begin{abstract}
Using the surface brightness fluctuations in HST WFPC-2 images, we determine that M87, NGC 4486B, and NGC 4478 are all at a distance of $\sim 16 \mathrm{Mpc}$, while NGC 4476 lies in the background at $\sim 21 \mathrm{Mpc}$. We also examine the globular clusters of M87 using archived HST fields. We detect the bimodal color distribution, and find that the amplitude of the red peak relative to the blue peak is greatest near the center. This feature is in good agreement with the merger model of elliptical galaxy formation, where some of the clusters originated in progenitor galaxies while other formed during mergers.
\end{abstract}

\section{Introduction}

An accurate estimate of the distance to M87 is important for the study of its other properties, in particular for determining the correct physical scales and luminosities from measured angular separations and aparent magnitudes. As the brightest, central galaxy in its portion of the Virgo cluster, M87 has a rich environment, including several companions and exeptionally large population of globular clusters (GC). By measuring accurate distances we can separate true neighbors from close projections, while mapping global properties of the GC population can give important clues for the merger history of M87 and the Virgo cluster in general.

Because of its smooth morphology, the surface brightness fluctuation (SBF) method of distance determination is ideally suited for application to M87. The method works by measuring the statistical effects of the galaxy being made up of a large number individual stars.

The basis for the method is clearest if one considers the idealized case of a galaxy with uniform surface brightness, whose stars all have the same luminosity, $l$. In an image of this galaxy, each pixel will contain the light from some number of stars, $n(x, y)$. The number of stars per pixel will have an average $\bar{n}$, and a standard deviation $\sqrt{\bar{n}}$. By examining the image, one can measure the mean flux in a pixel, $\bar{f}=\bar{n} l$, and its standard deviation, $\sigma_{f}=\sqrt{n} l$. Finally, one can use these to calculate the flux of a star in the galaxy: $l=\sigma_{f}^{2} / \bar{f}$. If we can estimate the absolute luminosity from this star, it may be used as a "standard candle," and the distance can be measured.

In practice, there are a number of complications. The morphology of the galaxy is not uniform, the variance due to stellar statistics must be separated 
from variance due to noise and contaminating objects, and galaxies are made up of stars with a variety of luminosities. All of these difficulties can be overcome to a large extent. A complete description of the process of SBF measurement can be found in the original paper by Tonry \& Schenider (1988), or in Neilsen, Tsvetanov, \& Ford (1997).

A second method of distance determination uses the globular cluster luminosity function (GCLF). Assuming that the luminosity function of globular clusters can be estimated, one may use it as a standard candle. It is particularly convenient to measure the GCLF on data for which one has already measured SBF, because it is necessary to locate the globular clusters in the image to obtain an SBF measurement. The creation of a catalog of globular clusters also allows for the study of the spatial and color distributions of the globular cluster population.

\section{The Data}

The Hubble Space Telescope archive provided all of the images used in this study. Figure 1 shows the placement of the fields around M87. For each field, we

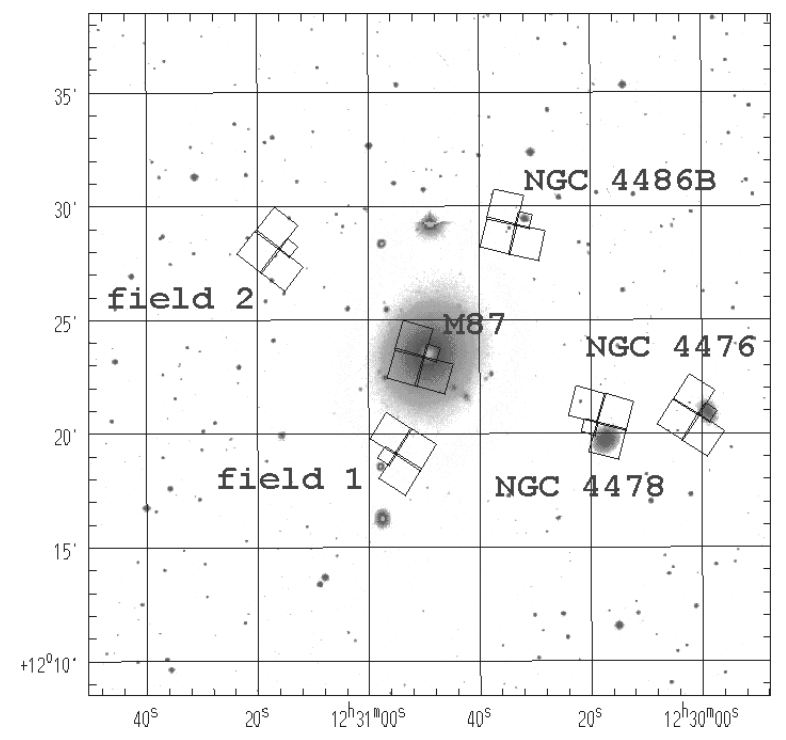

Fig. 1. The placement of the fields around M87. The image on which our fields are shown was created using an image from the GASP plates and an image taken by Hintzen et al (1993), supplied courtesy the NCSA Astronomy Digital Image Library.

used HST WFPC-2 images with a filter approximating the $I$ band (F814W) and a filter approximating the $V$ band (either F555W or F606W). Table 1 presents, for each field, the projected separation from the center of M87 to the center of the field, the filters used, the total exposure times, and the number of exposures in each filter. 


\begin{tabular}{llllll}
\hline \hline Field & Sep. (arcmin.) & Bands & Filters & $t_{\exp }$ (sec.) & \# exp. \\
\hline M87 center & 0.4 & V, I & F555W, F814W & 2400,2400 & 4,4 \\
field 1 & 4.5 & V, I & F606W, F814W & 3380,4200 & 5,4 \\
NGC 4486B 6.7 & V, I & F555W, F814W & 1800,2000 & 3,4 \\
field 2 & 8.2 & V, I & F606W, F814W & 1800,13400 & 3,5 \\
NGC 4478 & 8.2 & V, I & F606W, F814W & 16800,16500 & 6,6 \\
NGC 4476 & 12.0 & V, I & F555W, F814W & 2400,2400 & 4,4 \\
\hline
\end{tabular}

Table 1. The data used in this study.

\section{The Distances}

In table 2, we present the distances determined through our SBF and GCLF measurements. We also report the distance as determined by the planetary nebula luminosity function, as given by Ciardullo, Jacoby, \& Tonry (1993).

\begin{tabular}{lllll}
\hline \hline Method M87 & NGC 4486B & NGC 4478 & NGC 4476 \\
\hline SBF & $15.8 \pm 1.0$ & $16.2 \pm 1.0$ & $15.1 \pm 1.0$ & $21.1 \pm 1.1$ \\
GCLF & $14.2 \pm 1.7$ & & $16.4 \pm 3.7$ & $19.2 \pm 3.4$ \\
PNLF & $14.9 \pm 0.7$ & & & \\
\hline
\end{tabular}

Table 2. The distances to M87 and its companions in Mpc, by three methods.

To calculate the SBF distances, we measured the mean color in the utilized region of the galaxy using the two filters and converted to $V-I$ using the calibration of Holtzman et al. (1995), and the F814W SBF calibration of Ajhar et al. (1997). To calculate the GCLF distances, we estimate the peak of the GCLF using Ashman, Conti, \& Zepf (1995), whose theoretical results agree well with the observational calibration of Whitmore (1996). The distances measured by the different methods agree well. From these distances, it appears that NGC 4486B and NGC 4478 are genuine companions of M87, while NGC 4476 lies in the background.

\section{The Globular Cluster System of M87}

In four of our fields, (the center of M87, field 1, the NGC 4486B field, and field 2) we expect our globular cluster candidate catalog to be dominated by clusters from M87; this allows us to study the color distribution in several locations. It has been known for some time that the cluster population becomes bluer with distance from the center (see Strom et al. 1981), and a variety of explanations have been proposed. Strom et al. (1981) suggests that the clusters all formed at the same time, probably preceding the formation of the galaxy itself, and that the gradient in colors has the same origin as the gradient in the color of the integrated halo. In contrast, Ashman \& Zepf (1992) claim that M87 was formed as the result of mergers, which play a critical role in the formation of the globular cluster population. In this model, during mergers the red clusters are formed in 
the center of M87 from the gases in the merging galaxies, while the blue clusters, originally associated with the progenators, form the more extended cluster halo.

Figure 2 presents the color distributions in each field. In every field, there is a peak near $V-I=0.95$. The center field clearly shows a distinct peak near $V-I=1.2$ as well. There are traces of this second peak in field 1 and the in NGC 4486B field as well, but with smaller amplitude relative to the blue peak. It appears that there are two populations of globular clusters, the bluer of the two populations having a larger spatial extent. A Kolmogorov-Smirnov (K-S) test

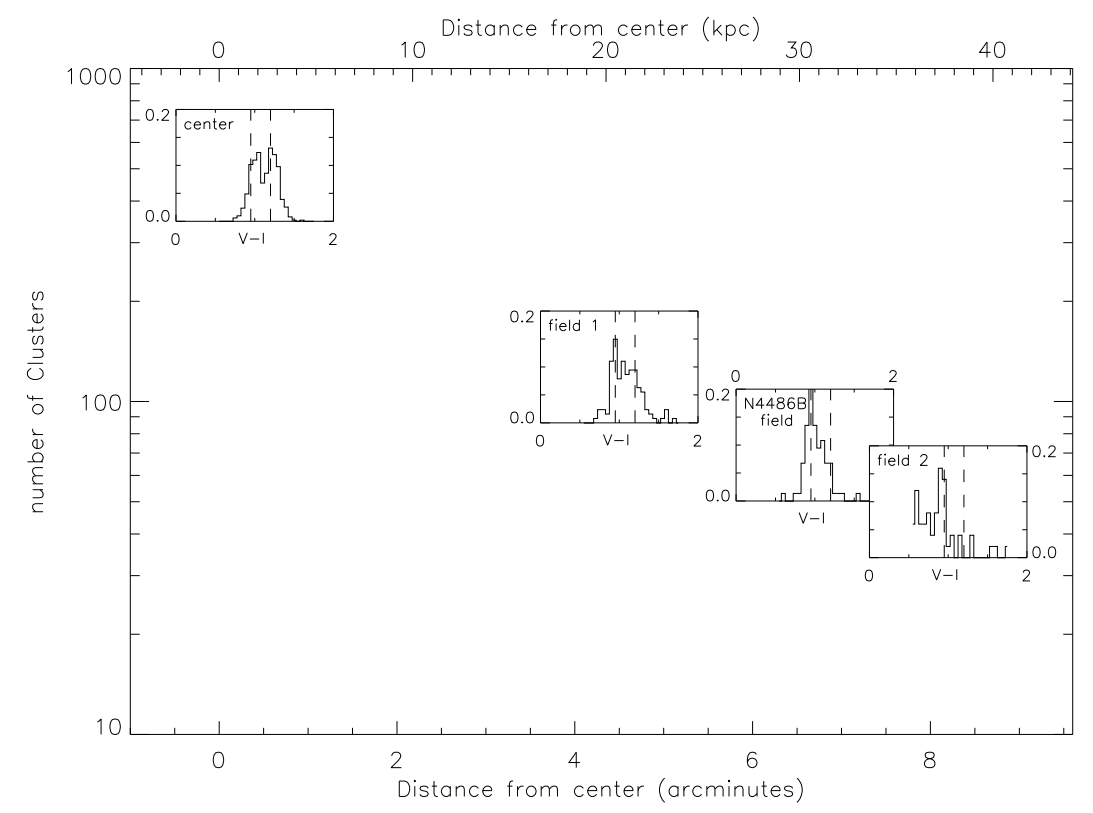

Fig. 2. The color distribution of M87's globular cluster in several fields. The placement of each plot represents the distance from the center of the field to the center of M87, and the total number of clusters with good $V-I$ colors data. The dashed lines are placed at $V-I=0.95$ and $V-I=1.20$.

on the 3 fields with reasonably good statistics rejects with a confidence of better then $97 \%$ that the data in each field arise from the same parent population, even if the different fields are shifted to have the same mean color. The data are consistent, however, with clusters in each of the fields arising from a double Gaussian distribution, supporting the a model where the central, red clusters are formed during mergers.

\section{Conclusions}

The surface brightness fluctuations and globular cluster luminosity functions allow us to measure the distances to M87 and several of its apparent neighbors, 
and determine which were true companions. Our globular cluster catalogs provide valuable information on the variation of the color distribution of globular clusters with the distance from the center. However, our statistics were insufficient to provide a more detailed understanding. In particular:

- M87, NGC 4486B, and NGC 4478 appear to be true companions, all about $16 \mathrm{Mpc}$ away. NGC 4476, on the other hand, is in the background, at approximately $21 \mathrm{Mpc}$.

- The K-S test on the globular cluster color distributions indicates that the samples detected at the different distances are unlikely to have arisen from the same population. We therefore conclude that the color distribution of the globular cluster population in M87 varies with distance.

- Even when the cluster populations are artificially shifted so that they have the same mean color, the K-S test still indicates that the different samples are unlikely to have arisen from the same population. This casts doubt on models where all clusters have the same origin.

- The color distribution in the central field is significantly better fit by two Gaussian distributions than one. Double peaked distributions with the peak widths and the difference in peak colors constrained to match the best fit double peaks of the center fit the other fields well. This result is consistent with current merger models.

\section{References}

Ajhar, E. A., Lauer, Tod R., Tonry, J. L., Blakeslee, J. P., Dressler, A., Holtzman, J. A., \& Postman, M., 1997, AJ, 114, 626.

Ashman, K. M., Conti, A., \& Zepf, S. E., 1995, AJ, 110, 1164.

Ashman, K. M., \& Zepf, S. E., 1992, ApJ, 384, 50.

Ciardullo, R., Jacoby, G. H., \& Tonry, J. L., 1993, ApJ, 419, 479.

Hintzen, P., Angione, R., Talbert, F., Cheng, K. P., Smith, E., \& Strecher, T. P., 1993, in NASA Ames Research Center, The Evolution of Glaxies and Their Environment. Holtzman, J. A., Burrows, C. J., Casertano, C., Hester, J. J., Trauger, J. T., Watson, A. M., \& Worthey, G., 1995, PASP, 107, 1065.

NCSA Astronomy Digital Image Library, http://imagelib.ncsa.uiuc.edu/imagelib

Neilsen, E. H., Jr., Tsvetanov, Z. I., \& Ford, H. C., 1997, ApJ, 483, 745.

Strom, S. E., Forte, J. C., Harris, W. E, Strom, K. M., Wells, D. C., \& Smith, M. G., 1981, AJ, 245, 416.

Tonry, J. L., \& Schneider, D. P., 1988, AJ, 96, 807.

Whitmore, B. C., in The Extragalactic Distance Scale, ed. M. Livio, M. Donahue, \& N. Panagiua (Cambridge: Cambridge Univ. Press). 